East African Medical Journal Vol. 80 No 10 October 2003

PREVALENCE OF HIV/AIDS AND PSYCHIATRIC DISORDERS AND THEIR RELATED RISK FACTORS AMONG ADULTS IN EPWORTH, ZIMBAMBWE M.B. Sebit, PhD., P. Maramba, Department of Psychiatry, University of Zimbabwe, Faculty of Medicine, P.O. Box A178, Avondale, Harare, Zimbabwe, M. Tombe, MMed (Med), S. Balus, MMed (Obst. \& Gynae.) and S.D.A. Nkomo, MD, Ministry of Health and Child Welfare, Zimbabwe

Request for reprints to: Dr. M.B. Sebit, Department of Psychiatry, Faculty of Medicine, University of Zimbabwe, P.O. Box A178, Avondale, Harare, Zimbabwe

\title{
PREVALENCE OF HIVIAIDS AND PSYCHIATRIC DISORDERS AND THEIR RELATED RISK FACTORS AMONG ADULTS IN EPWORTH, ZIMBAMBWE
}

\author{
M.B. SEBIT, M. TOMBE, S. SIZIYA, S. BALUS, S.D.A. NKOMO and P. MARAMBA
}

\begin{abstract}
Objectives: To examine the prevalence of HIV infection, neuropsychiatric disorders, psychiatric symptoms/signs, alcohol use/misuse, CD4 cell counts and risk factors in adult patients.

Design: Cross-sectional study.

Setting: Epworth, which is about $15 \mathrm{~km}$ on the southeastern part of Harare, Zimbabwe. Subjects: Two hundred subjects were included in the study out of which six were excluded beacause of HIV-1 indeterminate results.

Materials and Methods: A convenience sample of 200 subjects recruited in a crosssectional study in Epworth, Zimbabwe. Six subjects had indeterminate HIV-I antibody results and were excluded from the study. The remaining 194 subjects of whom 101 (52.1\%) knew about their sero-status and were consecutively recruited, whereas, 93 (47.9\%) did not know about their sero-status and were recruited by a systematic random sampling method (1-in-3). They were then interviewed about neuropsychiatric disorders using BPRS, MADRS, AUDIT and MINI Mental State Test, including the risk factors related to HIV infection. After ELISA tests' results, the two groups were combined and then categorised into HIV positive $(n=115)$ and HIV negative $(n=79)$ subjects. Main outcome measures: Prevalence, neuropsychiatric disorders, increased CD4 cell counts and risk factors associated with HIV infection.

Results: The findings were that the overall point prevalence of the HIV infection was $59.3 \%(115 / 194)$. Comparative analyses between seropositive and seronegative HIV/AIDS subjects showed: over two thirds $\mathbf{( 7 1 . 3 \% )}$ of the HIV positive subjects suffered from psychiatric disorders, more than those with HIV negative $44.3 \%(\mathrm{OR}=3.12,95 \% \mathrm{Cl}=$ 1.64-5.95, $P=0.0002$ ), and subjects aged 35 years and less were mostly HIV seronegatives $(n=77.2 \%, O R=2.34,95 \% \mathrm{CI}=\mathbf{1 . 1 8}-\mathbf{4 . 7 5}, \mathrm{P}=\mathbf{0 . 0 1 4})$. The overall prevalence of alcohol use/misuse was $41(21.1 \%)$, with higher prevalence rate among HIV positive subjects, $28(24.3 \%)$ than those who were HIV negative, $13(16.5 \%)$. The commonest psychiatric symptoms/signs $(P<0.05)$ were emotional withdrawal, depressed mood, suspiciousness, apparent sadness, reduced sleep and suicidal thoughts (specially among women).

Conclusion: There is very high point prevalence of HIV/AIDS and psychiatric disorders, including a moderate prevalence rate of alcohol use/misuse in this less affluent community that warranted intervention.
\end{abstract}

\section{INTRODUCTION}

In Zimbabwe there are very scanty studies done in the community pertaining to HIV/AIDS prevalence, neuropsychiatric disorders, substance use/misuse and risk factors. Studies that have been done in Zimbabwean rural areas seemed to indicate a wide range prevalence of HIV infection ranging from 22\%-26.3\%(1,2). While presence of various STDs has been associated with higher prevalence rates of HIV-I infection in many studies $(3,4)$, however, establishing how much the STD is a risk for transmission is difficult. It is of no doubt that the higher prevalence of STDs in developing countries helps in transmission of $\operatorname{HIV}(4,5)$. Besides, women with multiple sexual partners are highly associated with HIV infection (6). The proportion of cases attributed to heterosexual transmission has increased from $3 \%$ to $10 \%$ (7).

HIV/AIDS individuals are prone to a wide range of psychiatric disorders. Depression and psychosis among HIV infected patients are frequently being recognised by clinicians $(8,9)$. The rates of major depression range from $4 \%$ to $21 \%(10,11)$ compared with the total population lifetime rate of major depression, which is $3 \%$ (12). Mania may occur in up to $9 \%$ of patients over the course of their HIV illness 
and psychosis often emerges during later stages of AIDS(13). Anxiety disorders appear to occur with the same frequency as in the general population (14). Cognitive deficits among AIDS patients have long been described (15), but subsequent studies did not provide evidence of cognitive impairment $(16,17)$. Alcoholics may be at a higher risk for infection by HIV-I, because of their higher lifetime prevalence of practicing risky behaviour such as an intravenous drug use $(18,19)$. In addition to strong association between alcohol use and unsafe sexual practice among psychoactive substance users (20). The majority of drug users who are HIV infected may suffer from other psychiatric disorders $(21,22)$. It is suggested that the severity of psychiatric symptoms after HIV testing may be greater in drug users than non-drug users (23), consequently, drug use/ misuse is a more important cause of psychopathology than the virus itself (24).

At present most psychiatric disorders, and all the so-called functional disorders, are classified largely on the basis of their clinical symptoms, or, to be precise, a combination of the symptoms the patient complains of and the behavioural abnormalities evident on the history and mental state examination. A recent study by Sebit et al. (10) among HIV/AIDS depressed patients found the most significant symptoms/signs were lassitude, pessimistic thoughts and suicidal thoughts. Psychiatric symptomatology among HIV/ AIDS persons has been studied in Zimbabwe (10), but not among the HIV/AIDS seronegative individuals. Absolute CD4 Iymphocyte counts are still being used in monitoring the severity of immunologic damage in HIV infected persons $(10,25)$. The main research questions were two: (a) to what extent is the point prevalence of HIV infection in this less affluent Epworth community? and (b) are there any differences/ associations between those subjects with and without HIV/AIDS infections with respect to neuropsychiatric disorders, alcohol use/misuse, psychiatric symptoms/ signs, CD4 cell count, risk factors and socio-demographic variables?

\section{MATERIALS AND METHODS}

The study was conducted in Epworth, which is about $15 \mathrm{Km}$ on the Southeastern of Harare, Zimbabwe. According to the 1992 census by Central Statistics Office (CSO) (26), the Epworth health indicators seemed to suggest that the growth rate was estimated at $10 \%$ and a birth rate at $4.1 \%$. About $50 \%$ of the people were formally employed, but mostly unskilled labourers. The common health hazards prevailing in this area were over crowdness, poor sanitation and other unhealthy behaviours (26).

Participants: The study's design was a cross-sectional study, using a convenient sampling size of 200 subjects. Six out of 200 subjects were excluded from the analysis, because of the HIV-I indeterminate results. For a practical reason, since the primary aim of this study was to determine the point prevalence of HIV/AIDS in this less affluent community, it was therefore justifiable to use two sample populations and two sampling methods for subjects' recruitment. Participation was voluntary. The first sample population comprised of 101 $(52.1 \%)$ subjects who claimed to know about their serostatus of being HIV/AIDS seropositive and was consecutively recruited. In this group, those who had mental illness, prior neurological disease, head trauma, being a pregnant woman or seriously sick were excluded from the study. The second sample population comprised of $93(47.9 \%)$ subjects who did not know about their HIV serostatus and were recruited by a systematic random sampling (1-in-3 houses) of a population of houses. Any eligible subject in that house was recruited for the study. In this second group, eligible subjects were those who had no history of TB, hypertension, diabetes mellitus and mental illness. Both groups were recruited from the same areas of the Epworth and subjects aged 18-55 years were included in the study. Below the age of eighteen years, subjects were excluded from the study, because of the consent and above the fifty-five years to avoid including subjects with possible degenerative diseases. Both male and female subjects were included. Since the secondary aim of this study was to determine the prevalence of neuropsychiatric disorders, alcohol use/misuse, psychiatric symptoms/signs and risk factors in relation to the serostatus of the subjects, the two groups were combined and re-categorised after the ELIZA results for HIV-I antibody into HIV positive $(n=115)$ and HIV negative $(\mathrm{n}=79)$ subjects.

Assessment instruments: Socio-demographic characteristics and the use of antiretroviral drugs or any other drugs were obtained from the subjects. A particular attention was paid to collecting data on number of sex partners in the last five years, STD, homosexual or lesbianism preference. The module used for psychiatric assessment included the sections for psychotic, affective, and anxiety disorders of a modified version of the Composite International Diagnostic Interview (CIDI) (27), which incorporates algorithms for the psychiatric diagnosis according to the DSM-IV (28). CIDI comprised of two modules. The first module was the Brief Psychiatric Rating Scale $(29,30)$ (BPRS), which had an 18item dimension rating scale filled out on a patient based on a semi-structured interview. Each dimension represents a domain of psychiatric symptomatology and is rated on a seven-point Likert Scale from "not present $=0$ ", "very mild= 1", "Mild = 2", "Moderate = 3", "Moderately severe $=4$ " to "extremely severe $=6$ " and "uncertained or not applicable $=9 "$. It provides a quantitative score that reflects global pathology and incorporates algorithms for the psychiatric diagnosis according to the DSM-IV (28). The second module was the Montgomery-Asberg Depression Rating Scale (MADRS) (31) used to assess depressive symptoms in HIV/AIDS subjects. It is a 10-item questionnaire that measures the frequency of depressive symptoms at a six-point scale. Responses for each item range from none of the time or not present (scored as 0 ) to most or all of the time (scored as 6) or not applicable or uncertain (scored 9). Summing across the 10-items of the MADRS that give score of at least 20 on the MADRS means presence of prominent depressive symptoms. Mini Mental State Test (32) is a quick test used to get a rough index of cognitive impairment. It assessed orientation, registration, attention/concentration, recall, language, and construction. It takes five minutes to administer. It consists of thirty points' scores, a score of less than 25 suggests impairment, but a score of less than 20 usually suggests definite impairment. Karnofsky Performance Scale (KPS) (33) is a summary of clinical status of a patient based on a physical functional index ranging from zero to 100 . A score of zero means death and 100 suggests normality. Cases were considered positive 
by the Centre for Disease Control and Prevention (34) case definitions for ATDS if an indicator disease was present after thorough clinical interviews and physical examinations using a questionnaire.

Subjects were interviewed using the Alcohol Use Disorders Identification Test (AUDIT) $(35,36)$, instrument. The AUDIT was devised by World Health Organisation (WHO) as a brief screening method suitable for identification of currently active hazardous and harmful drinkers in general medical settings in both developing and developed countries. The 10 item AUDIT questionnaire measured alcohol consumption, dependence symptoms, personal and social harm reflective of drinking. Cut off scores for identifying cases with evidence of alcohol-related problems were scores of $<16$ points for hazardous drinkers and $\geq 16$ points for hazardous drinkers and $\geq 16$ for harmuful drinkers in the AUDIT instrument. A modified questionnaire developed by Duke et al. (37) was used to assess about life time and current (last 12 months) use of cannabis, amphetamine, cocaine, crack, opiates, LSD, ecstasy, barbiturates, benzodiazepine solvent, including mudzepete and tobacco. Mudzepete is a psychostimulant substance used in Zimbabwe by snuffing. AUDIT rated the frequency of use as every day, 1-3 times a week, less than once a week but more than once a month, less than once a month, and never.

Procedure: The Medical Research Council of Zimbabwe approved the study protocol (MRCZ). After written informed consent was obtained, eligible subjects were enrolled for the study. Six Village Community Workers (VCWs) helped in mobilising subjects from the community. Each VCW was assigned to mobilise and assemble subjects in one house for clinical interviews and physical examinations, including weighing, measuring the blood pressure and taking of whole blood specimens. Each subject received pre-test counseling before the whole blood was obtained for diagnostic laboratory tests. Those who wanted their HIV tests' results were provided with post-test counseling. The language used in the study was Shona. A professional translator translated the questionnaires into Shona language. The research team consisted of General Practitioners and specialists and was trained using a WHO Training Manual (38) in a series of a live examination of HIV-I seropositive subjects in the community before running the main study. There was a spot check for conformity to protocol throughout the study period. Patients were assigned on the bases of clinical evaluation and investigation to one of three groups of the CDC (34) criteria for AIDS staging.

Laboratory investigations: The blood samples were collected in EDTA vacutainer tubes that contained tripotassium ethylene diamine tetra-acetate (K3EDTA) anticoagulant. The Genelavia mix assessed the HIV-I and II serostatus (Sanofi Diagnostics Pasteur, France), and Recombigen (Cambridge Diagnostics, Galway, Ireland) HIVI and II enzyme immunoassay Kits. CD4 and CD8 positive Iymphocytes were visualised and enumerated according to the method of Lisse et al (39), as modified in WHO manual (40). The absolute CD4 (CD) count was determined as WBC X lymphocyte \% X CD4 (CD8)/200. The Iymphocyte percentage and WBC values were derived from Coulter counts.

Statistical analysis: Two levels of statistical analyses were carried out using SPSS/PC + software (SPSS Inc., Chicago, Illinois, USA). The first level of analysis was to get the overall prevalence of HIV infection; whereas, the second level of analysis were the comparisons between those who were HIV positive and HIV negative. Non-parametric tests were used for group comparisons (HIV-I seropositive versus HIV-I seronegative subjects) using the $\mathrm{X}^{2}$ test, with Yates corrected P-value. When expected values were five or below Fisher's exact 2-tailed values of P-value and analysis exact confidence intervals were calculated. Psychiatric diagnoses were made according to the DSM-IV, with the total scores on the individual items of the BPRS/ MADRS. Summary statistics of change in CD4 counts using Wilcoxon test was done. ANOVA test was used for KPS and weight to compare between serostatus groups (HIV positive versus HIV positive) as a dependent variable. Ninenty five percent confidence intervals for the differences between means, $\mathrm{F}$ statistics and significance were set at a $5 \%$.

\section{RESULTS}

The overall prevalence of HIV infection was $59.3 \%$ (115/194). However, the HIV/AIDS prevalence was higher among subjects who claimed to be HIV seropositive, $80.2 \%$ (81/101) than among those who did not know about their serostatus, 36.6\% (34/93). Figure 1 , shows that those subjects who knew about their serostatus were 7.03 times more likely to be HIV seronegative than those who were HIV-I seropositive ( $\mathrm{OR}=7.03,95 \% \mathrm{CI}=3.52-14.18 ; \mathrm{P}<0.0001)$. Comparisons between HIV positive and HIV negative subjects after re-categorisation of the subjects showed that the HIV positive subjects' CDC (38) diagnoses as follows: asymptomatic, 54 (47.0\%), PGL, 33 (28.7\%), and AIDS, $28(24.3 \%)$ cases (Figure 2$)$. The mean \pm s.d. weight being higher for HIV seronegative subjects, 59.8 \pm 8.3 than HIV seropositive subjects, $55.3 \pm 10.9$ (Fstatistic 7.9, $\mathrm{P}=0.006)$. The mean \pm s.d. KPS's scores being higher for HIV seronegative subjects, $104.5 \pm$ 80.2 than for HIV seropositive subjects, $87.2 \pm 11 . \overline{6}$, (F-statistic 5.2, P=0.024). This means that the HIV seronegative subjects had better weights and healthier than those with HIV/AIDS. ANOVA showed the HIV seropositive subjects were older, mean \pm s.d. $(33.3 \pm$ 8.5) than those who were HIV seronegatives (28.7 \pm 9.95), $\mathrm{P}=0.001$. It can be seen in Table 1 that subjects aged 35 years and less were 2.34 times more likely to be HIV seronegative than those aged 36 years and above $(\mathrm{n}=77.2 \%, \mathrm{OR}=2.34,95 \% \mathrm{CI}=1.184 .75, \mathrm{P}=$ 0.014 ), otherwise no significant differences were found between HIV positive versus HIV negative with regard to gender, educational attainment and occupation. However, the majority of the subjects $(62.0 \%)$ were married/cohabited $(\mathrm{P}<0.0001)$ and were mostly HIV seronegative.

It can be seen in Table 2 that over two thirds $(71.3 \%)$ of the subjects who tested HIV seropositive suffered from mental illness $(\mathrm{OR}=3.12$, 95\% CI= 1.645.95, $(\mathrm{P}=0.0002)$ according to the BPRS. However, $68.4 \%$ of the subjects who were HIV-I seronegative had no depression $(\mathrm{OR}=2.91,95 \% \mathrm{CI}=1.53-5.57, \mathrm{P}=0.001)$ according to MADRS. DSM-IV psychiatric diagnoses as follows (i.e., HIV-seropositive versus HIV seronegative subjects): schizophrenia (18 versus 8), 
depression (27 versus 8), mania (1 versus 3), generalised anxiety disorders (19 versus 10$)$, and other psychiatric diagnoses (17 versus 6). Most of the subjects whether they were HIV seropositive or seronegative scored more than 25 points of having no cognitive impairments $(\mathrm{P}=0.017)$ in Mini Mental State Tests. Only five (6.3\%) subjects scored less than 20 points for a definitive cognitive impairment and were HIV seronegative. Table 3 shows significant differences were found related to risk factors: most of the HIV seronegative subjects $(62 \%)$ had a single partner $(\mathrm{P}=0.017)$ and $81 \%$ of them had no history of STD $(\mathrm{P}<0.0001)$. When we controlled for HIV serostatus and matched sex by number of partners, marital status or STD. The likelihood ratios were significant among HIV seronegative females who had single partners $(\mathrm{P}=0.012)$ and were married/ cohabited $(\mathrm{P}<0.0001)$. However, the likelihood ratio was significant $(\mathrm{P}=0.020)$ for HIV seropositive females who had two or more episodes of STD. No significant differences were found regarding sexual desire and blood transfusion (Table 3). None of the subjects were on anti-retroviral drugs. Table 4 shows CD 4 and CD 8 cell count: a significant difference was found between HIV seropositive and seronegative subjects as for CD4 cells count. As expected the Wilcoxon analysis showed a very high mean rank (82.4) for HIV-I seronegative subjects (significance, 2-tailed $\mathrm{P}<0.0001$ ) than seropositive subjects (51.0).
Figure 1

Comparison between group 1 and group 2

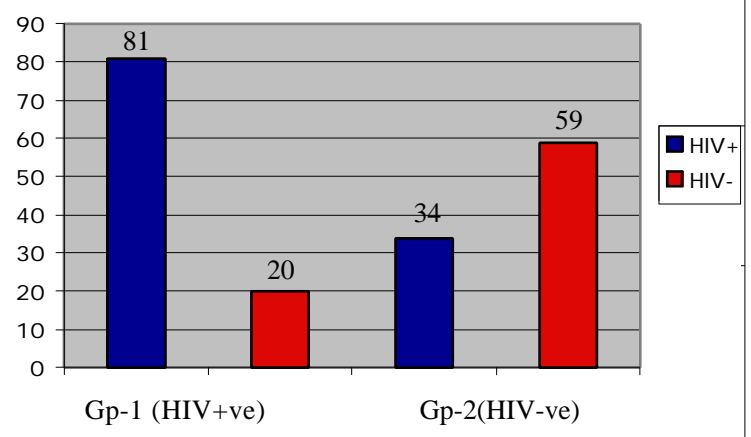

Figure 2

CDC diagnoses of the HIV seropositive

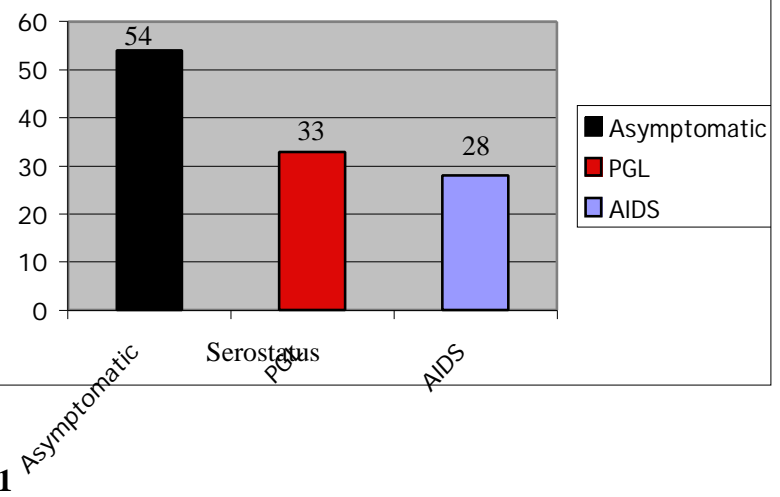

Socio-demographic characteristics of HIV/AIDS subjects

\begin{tabular}{|c|c|c|c|c|c|}
\hline Variable & $\begin{array}{l}\text { HIV negative }(\mathrm{n}=79) \\
\text { No. }(\%)\end{array}$ & $\begin{array}{l}\text { HIV positive }(\mathrm{n}=115) \\
\text { No. }(\%)\end{array}$ & OR & $95 \% \mathrm{CI}$ & P-value \\
\hline \multicolumn{6}{|l|}{ Age } \\
\hline$\leq 35$ & $61(77.2)$ & $68(59.1)$ & 2.34 & $1.18-4.75$ & $0.014^{*}$ \\
\hline $36+$ & $18(22.8)$ & 47 (40.9) & & & \\
\hline \multicolumn{6}{|l|}{ Sex } \\
\hline Male & $22(27.8)$ & $25(21.7)$ & 1.39 & $0.68-2.84$ & 0.421 \\
\hline Female & $57(72.2)$ & $90(78.3)$ & & & \\
\hline \multicolumn{6}{|l|}{ Marital status } \\
\hline Single & $18(22.8)$ & $5(4.3)$ & & & $<0.0001 *$ \\
\hline Married/coh. & $49(62.0)$ & $62(53.9)$ & & & \\
\hline Others & $12(15.2)$ & 48 (41.7) & & & \\
\hline \multicolumn{6}{|l|}{ Education } \\
\hline Nil & $4(5.1)$ & $8(7.0)$ & & & 0.754 \\
\hline Primary & $37(46.8)$ & $57(49.6)$ & & & \\
\hline Higher/Un./Col & $38(48.1)$ & $50(43.4)$ & & & \\
\hline \multicolumn{6}{|l|}{ Occupation } \\
\hline Nil & $45(57.0)$ & $52(45.2)$ & 1.60 & $0.87-2.98$ & 0.144 \\
\hline Employed & $34(43.0)$ & $63(54.8)$ & & & \\
\hline
\end{tabular}

$\mathrm{Coh}=$ cohabited $; \mathrm{Un} / \mathrm{Col}=$ University $/$ College $; * \mathrm{P}=$ significance 
Table 2

Distribution of neuropsychiatric disorders in HIV/AIDS subjects

\begin{tabular}{llllll}
\hline Variable & $\begin{array}{l}\text { HIV negative }(\mathrm{n}=79) \\
\text { No. }(\%)\end{array}$ & $\begin{array}{l}\text { HIV positive(n=115) } \\
\text { No. }(\%)\end{array}$ & OR & 95\% Cl & P-value \\
\hline BPRS & & & & & \\
$\quad$ No & $44(55.7)$ & $33(28.7)$ & 3.12 & $1.64-5.95$ & $0.0002^{*}$ \\
$\quad$ Yes & $35(44.3)$ & $82(71.3)$ & & & \\
MADRS & $54(68.4)$ & $49(42.6)$ & & $1.53-5.57$ & $0.001 * *$ \\
$\quad$ No & $25(31.6)$ & $66(57.4)$ & & \\
$\quad$ Yes & $5(6.3)$ & 0 & & & \\
MINI M. S. & $2(2.5)$ & $6(5.2)$ & & & \\
$\quad<20$ scores & $109(94.8)$ & & & \\
$\quad<25$ scores & & & & \\
$25+$ scores & $72(91.1)$ &
\end{tabular}

$* \mathrm{P} \& \# \mathrm{P}=$ significance

Table 3

Risk factors in HIV/AIDS infection

\begin{tabular}{|c|c|c|c|c|c|}
\hline Variable & $\begin{array}{l}\text { HIV negative }(n=79) \\
\text { No. }(\%)\end{array}$ & $\begin{array}{l}\text { HIV positive }(\mathrm{n}=115) \\
\text { No. }(\%)\end{array}$ & $x^{2}$ & Df & P-value \\
\hline \multicolumn{6}{|c|}{ Number of sexual Partners } \\
\hline Nil & 11(13.9) & $7(6.1)$ & 8.2 & 2 & $0.017 *$ \\
\hline One & $49(62.0)$ & $60(52.2)$ & & & \\
\hline $2+$ & $19(24.1)$ & $48(41.7)$ & & & \\
\hline \multicolumn{6}{|l|}{ STD } \\
\hline Nil & $64(81.0)$ & $55(47.8)$ & 22.5 & 2 & $<0.0001 * *$ \\
\hline Once & $10(12.7)$ & $31(27.0)$ & & & \\
\hline $2+$ & $5(6.3)$ & $29(25.2)$ & & & \\
\hline \multicolumn{6}{|l|}{ Sexual desire } \\
\hline None & $72(91.1)$ & $113(98.2)$ & & & \\
\hline Homosexual & 0 & $1(0.9)$ & & & \\
\hline Lesbian & $7(8.9)$ & $1(0.9)$ & & & \\
\hline \multicolumn{6}{|l|}{ Blood transfusion } \\
\hline No & $72(91.1)$ & $103(89.6)$ & & & 0.907 \\
\hline Yes & $7(8.9)$ & $12(10.4)$ & & & \\
\hline
\end{tabular}

$* \mathrm{P}=$ significance

Table 4

Distribution of CD4 and CD8 in relation to HIV/AIDS

\begin{tabular}{|c|c|c|c|c|c|}
\hline Variable & $\mathrm{N}$ & $\begin{array}{l}\text { Mean } \\
\text { Rank }\end{array}$ & Wilcoxon & $\mathrm{Z}$ & $\begin{array}{c}\text { Asymp sig } \\
\text { (2-tailed) }\end{array}$ \\
\hline \multicolumn{6}{|l|}{ CD4 cells count } \\
\hline HIV negative & 55 & 85.4 & 3725.0 & -4.7 & $<0.0001 *$ \\
\hline HIV positive & 75 & 51.0 & & & \\
\hline \multicolumn{6}{|l|}{ CD8 cells count } \\
\hline HIV negative & 24 & 35.8 & 859.0 & -0.60 & 0.547 \\
\hline HIV positive & 51 & 39.0 & & & \\
\hline
\end{tabular}

\footnotetext{
* $\mathrm{P}=$ significance
} 
Table 5

Socio-demographic characteristics of the alcoholics

\begin{tabular}{|c|c|c|c|c|c|c|c|}
\hline \multirow[t]{3}{*}{ Variable } & \multicolumn{2}{|c|}{ HIV positive } & \multicolumn{2}{|c|}{ HIV negative } & \multirow[t]{3}{*}{ OR } & \multirow[t]{3}{*}{$95 \% \mathrm{CI}$} & \multirow[t]{3}{*}{ P-value } \\
\hline & Non-Users $(\mathrm{n}=87)$ & Users $(\mathrm{n}=28)$ & Non-Users $(n=66)$ & Users $\quad(n=13)$ & & & \\
\hline & No. $(\%)$ & No. $(\%)$ & No. $(\%)$ & No. $(\%)$ & & & \\
\hline \multicolumn{8}{|l|}{ Age (years) } \\
\hline $16-25$ & $18(20.7)$ & $6(21.4)$ & $29(44.0)$ & $8(61.5)$ & 1.21 & $0.29-4.7$ & 0.996 \\
\hline $26-35$ & $35(49.2)$ & $9(32.2)$ & $22(33.3)$ & $2(15.4)$ & 2.82 & $0.5-28.9$ & 0.305 \\
\hline $36+$ & $3(39.1)$ & $13(46.4)$ & $15(22.7)$ & $3(23.1)$ & 1.91 & $0.4-11.8$ & 0.523 \\
\hline \multicolumn{8}{|l|}{ Gender } \\
\hline Male & $13(14.9)$ & $12(42.9)$ & $15(22.7)$ & $7(53.8)$ & 1.98 & $0.52-78$ & 0.406 \\
\hline Female & $74(85.1)$ & $16(57.1)$ & $51(77.3)$ & $6(46.2)$ & 1.84 & $0.63-6.1$ & 0.335 \\
\hline \multicolumn{8}{|l|}{ Marital Status } \\
\hline Single & $2(2.3)$ & $3(10.7)$ & $12(18.2)$ & $6(46.2)$ & 3.00 & $0.2-43.1$ & 0.343 \\
\hline Married/Coh. & $50(57.5)$ & $12(42.9)$ & $44(66.7)$ & $5(38.5)$ & 2.11 & $0.63-8.2$ & 0.287 \\
\hline Others* & $35(40.2)$ & $13(46.4)$ & $10(15.1)$ & $2(15.3)$ & 1.86 & $0.3-19.5$ & 0.712 \\
\hline \multicolumn{8}{|l|}{ Education } \\
\hline Nil & $10(11.5)$ & $5(17.8)$ & $6(9.1)$ & 0 & & & 0.262 \\
\hline $1-7$ years & $45(51.7)$ & $12(42.9)$ & $31(47.0)$ & $6(46.2)$ & 1.38 & $0.42-4.9$ & 0.754 \\
\hline $8+$ & $32(36.8)$ & $11(39.3)$ & $29(43.9)$ & $7(53.8)$ & 1.42 & $0.43-4.9$ & 0.705 \\
\hline \multicolumn{8}{|l|}{ Occup. } \\
\hline Nil & $17(19.5)$ & $9(32.2)$ & $18(27.3)$ & $5(38.2)$ & 1.91 & $0.45-8.7$ & 0.497 \\
\hline Employed & $37(42.5)$ & $16(57.1)$ & $17(25.8)$ & $4(30.8)$ & 1.84 & $0.48-8.6$ & 0.495 \\
\hline Others** & $33(38.0)$ & $3(10.7)$ & $31(46.9)$ & $4(30.8)$ & 0.70 & $0.10-4.5$ & 0.710 \\
\hline
\end{tabular}

Coh. = cohabited; Occup. $=$ occupation; Others $*=$ divorced $/$ widowed/separated, Others** $=$ house wife, self-employed, students

Table 6

Audit score and other substance use/misuse versus serostatus

\begin{tabular}{llllll}
\hline Score & $\begin{array}{l}\text { HIV positive }(\mathrm{n}=115) \\
\text { No. }(\%)\end{array}$ & $\begin{array}{l}\text { HIV positive }(\mathrm{n}=79) \\
\text { No. }(\%)\end{array}$ & $\mathrm{X}^{2}$ & df & P-value \\
\hline $\begin{array}{l}\text { Alcohol } \\
\quad \text { Nil }\end{array}$ & & & & \\
$\quad$ Scores <16 & $197(75.7)$ & $66(83.5)$ & 1.76 & 2 & 0.415 \\
$\quad$ Scores 16+ & $9(7.8)$ & $9(11.4)$ & & \\
Other substances & $4(5.1)$ & & \\
$\quad$ Cannabis & $4(3.5)$ & $1(1.3)$ & & \\
$\quad$ Tobacco & $23(20)$ & $5(6.3)$ & & \\
Mudzepete & $3(2.6)$ & $1(1.3)$ & \\
\hline
\end{tabular}

Table 7

Comparison of depression, risk factors, HIV positive status and alcoholism

\begin{tabular}{|c|c|c|c|c|c|c|c|}
\hline \multirow[t]{3}{*}{ Variable } & \multicolumn{2}{|c|}{ HIV positive } & \multicolumn{2}{|c|}{ HIV negative } & \multirow[t]{3}{*}{ OR } & \multirow[t]{3}{*}{$95 \% \mathrm{CI}$} & \multirow[t]{3}{*}{ P-value } \\
\hline & Non-users & 37) $\operatorname{Users}(n=28)$ & Non-Us & 6) $\operatorname{Users}(n=13)$ & & & \\
\hline & No. $(\%)$ & & No. $(\%)$ & No. $(\%)$ & & & \\
\hline \multicolumn{8}{|c|}{$\begin{array}{l}\text { Number of sexual } \\
\text { partners }\end{array}$} \\
\hline Nil & $7(8.0)$ & 0 & $10(15.2)$ & $1(7.7)$ & Und & Und & 1.000 \\
\hline One & $50(57.5)$ & $10(35.7)$ & $44(66.7)$ & $5(38.5)$ & 0.57 & $0.14-2.0$ & 0.487 \\
\hline $2+$ & $30(34.5)$ & $18(64.3)$ & $12(18.1)$ & $7(53.8)$ & 0.97 & $0.27-3.3$ & 0.818 \\
\hline \multicolumn{8}{|l|}{ STD } \\
\hline Nil & $43(49.4)$ & $12(42.9)$ & $54(81.8)$ & $10(76.9)$ & 0.66 & $0.23-1.9$ & 0.528 \\
\hline Once & $22(25.3)$ & $9(32.1)$ & $8(12.1)$ & $2(15.4)$ & 0.16 & $0.05-4.1$ & 0.700 \\
\hline $2+$ & $22(25.3)$ & $7(25.0)$ & $4(6.1)$ & $1(7.7)$ & 0.79 & $0.01-9.9$ & 1.000 \\
\hline \multicolumn{8}{|l|}{ MADRS } \\
\hline Depression & $53(60.9)$ & $13(46.4)$ & $19(28.8)$ & $6(46.2)$ & 1.29 & $0.35-4.3$ & 0.871 \\
\hline Nil & $34(39.1)$ & $15(53.6)$ & $47(71.2)$ & $7(53.8)$ & 0.34 & $0.11-1.0$ & 0.052 \\
\hline
\end{tabular}


Table 8

Socio-demographic characteristics of HIV/AIDS subjects

\begin{tabular}{|c|c|c|c|c|c|}
\hline Variable & $\begin{array}{l}\text { HIV positive }(\mathrm{n}=79) \\
\text { No. }(\%)\end{array}$ & $\begin{array}{l}\text { HIV negative }(\mathrm{n}=115) \\
\text { No. }(\%)\end{array}$ & OR & $95 \% \mathrm{CI}$ & P-value \\
\hline \multicolumn{6}{|l|}{ Age (years) } \\
\hline$\leq 35$ & $61(77.2)$ & $68(59.1)$ & 2.34 & $1.18-4.75$ & $0.014 *$ \\
\hline $36+$ & $18(22.8)$ & $47(40.9)$ & & & \\
\hline \multicolumn{6}{|l|}{ Sex } \\
\hline Male & $22(27.8)$ & $25(21.7)$ & 1.39 & $0.68-2.84$ & 0.421 \\
\hline Female & $57(72.2)$ & $90(78.3)$ & & & \\
\hline \multicolumn{6}{|l|}{ Marital status } \\
\hline Single & $18(22.8)$ & $5(4.3)$ & & & $<0.0001 *$ \\
\hline Married/coh. & $49(62.0)$ & $62(53.9)$ & & & \\
\hline Others & $12(15.2)$ & $48(41.7)$ & & & \\
\hline \multicolumn{6}{|l|}{ Educatoin } \\
\hline Nil & $4(5.1)$ & $8(7.0)$ & & & 0.754 \\
\hline Primary & $37(46.8)$ & $57(49.6)$ & & & \\
\hline Higher/Un./Col. & $38(48.1)$ & $50(43.4)$ & & & \\
\hline \multicolumn{6}{|l|}{ Occupation } \\
\hline Nil & $45(57.0)$ & $52(45.2)$ & 1.60 & $0.87-2.98$ & 0.144 \\
\hline Employed & $34(43.0)$ & $63(54.8)$ & & & \\
\hline
\end{tabular}

Coh. $=$ cohabited, Un./Col. $=$ University/College, $* \mathrm{P}=$ significance

Table 9

Significant findings according to the Brief Psychiatric Rating Scale (BPRS) for the assessment of psychiatric symptoms

\begin{tabular}{|c|c|c|c|c|c|}
\hline \multirow{2}{*}{$\begin{array}{l}\text { Symptom } \\
\text { Somatic concern }\end{array}$} & \multirow{2}{*}{$\begin{array}{l}\text { HIV positive } \\
\text { Mean rank } \\
111.1\end{array}$} & \multicolumn{2}{|c|}{$\begin{array}{l}\text { HIV negative MWU } \\
\text { Mean rank }\end{array}$} & \multirow{2}{*}{$\begin{array}{l}\mathrm{Z} \\
-4.2\end{array}$} & \multirow{2}{*}{$\begin{array}{l}\text { Significance } \\
\text { 2-tailed } \\
0.000\end{array}$} \\
\hline & & 77.7 & 2977 & & \\
\hline Anxiety & 107.9 & 82.4 & 3346 & -3.2 & 0.001 \\
\hline Emotional withdrawal & 112.5 & 75.7 & 2816.5 & -4.8 & 0.000 \\
\hline Coceptual disorganization & 108.6 & 81.3 & 3264.5 & -3.7 & 0.000 \\
\hline Tension & 106.1 & 84.9 & 3550.5 & -2.7 & 0.007 \\
\hline Depressed Mood & 107.9 & 82.3 & 3341.5 & -3.2 & 0.001 \\
\hline Hostility & 105.2 & 86.4 & 3663 & -2.4 & 0.017 \\
\hline Suspiciousness & 107.1 & 83.5 & 3437 & -3.4 & 0.001 \\
\hline Hallucinatory behaviour & 102.4 & 90.3 & 3976.5 & -1.96 & 0.050 \\
\hline Motor retardation & 90.7 & 107.33 & 3765.5 & -2.6 & 0.009 \\
\hline Unusual Thought content & 104.4 & 87.5 & 3755 & -2.8 & 0.006 \\
\hline
\end{tabular}

MWU= Mann-Whitney U Test

\section{Table 10}

HIV prevalence and depressive symptoms/signs by gender

\begin{tabular}{llll}
\hline Variable & $\begin{array}{l}\text { Likehood ration } \\
\text { value }\end{array}$ & d.f & P-value \\
\hline $\begin{array}{lll}\text { Apparent sadness } \\
\quad \text { Male }\end{array}$ & 10.2 & 4 & $0.037^{*}$ \\
$\quad$ Female & 21.3 & 6 & 0.002 \\
$\begin{array}{l}\text { Reported sadness } \\
\text { Male }\end{array}$ & 19.9 & 3 & $<0.0001^{*}$ \\
$\quad$ Female & 10.3 & 6 & 0.112 \\
Inner tension & 12.4 & 5 & $0.030^{*}$ \\
$\quad$ Male & 6.15 & 5 & 0.292 \\
$\quad$ Female & 6.7 & 2 & $0.013^{*}$ \\
Inability to feel & 6.8 & 4 & 0.144 \\
$\quad$ Male & & 2 & 0.843 \\
$\quad$ Female & 6.3 & 4 & $0.017^{*}$ \\
Apparent sadness & 12.0 & & \\
$\quad$ Male & & & \\
$\quad$ Female & & & \\
\hline
\end{tabular}

* $\mathrm{P}=$ significance 
Alcohol use/misuse: The overall point prevalence of alcohol use/misuse among subjects was $21.1 \%(n=41$; $95 \% \mathrm{CL}=15.4-26.8)$. Table 5 shows that there were no significant differences found between alcohol users versus non-users and socio-demographic factors with regard to the HIV/AIDS prevalence. However, further analyses were done among HIV/AIDS subjects only with regard to alcohol users versus non-users: females were 0.23 times more likely to be non-users of alcohol $(\mathrm{n}=74, \mathrm{OR}=0.23,95 \% \mathrm{CI}=0.08-0.68, \mathrm{P}=0.004)$, likewise, HIV/AIDS subjects were mostly employed and users/ misusers of alcohol $\left(n=16, X^{2}=7.40, d f=2, P=0.024\right)$. AUDIT scores in Table 6 show there was no significant difference between those with and without HIV infection, when comparisons were made between users and nonusers of $(\mathrm{P}=0.415)$ or even between hazardous and harmful drinkers $(\mathrm{P}=0.253)$. But there was a slightly high prevalence rate of alcohol use/misuse among the HIV positive subjects, $24.3 \%$ (28/115) than those who were HIV negative, and $16.5 \%$ (13/79). Other substance of use, tobacco was the most used substance, $20 \%$. Table 7 shows no significant differences were found between HIV serostatus and alcohol status of the subjects with regard to number of sexual partners, a history of STD or depression. However, a significant number of subjects $(71.2 \%)$ had no depression and were non-users of alcohol $(\mathrm{P}=0.052)$. On a further analysis made among HIV seropositive subjects versus their alcohol status (users versus non-users) a significant difference was found between those who had one versus two partners $(\mathrm{OR}=3.00,95 \% \mathrm{CI}=1.13-8.28$, $\mathrm{P}=0.025)$ with $64.3 \%$ of those who had two or more partners used/misused alcohol.

Psychiatric symptoms/signs: A non-parametric analysis was used, Mann-Whitney U test, to compare between subjects serostatus and individual psychiatric symptoms/signs (Table 8 and 9). Only eleven out of the 19 BPRS's symptoms/signs showed significant $(\mathrm{P}<0.05)$ mean rank differences in terms of the HIVI seroprevalence: Somatic concern, anxiety, emotional withdrawal, conceptual disorganisation, tension, depressed mood, hostility, suspiciousness, hallucinatory behaviour, motor retardation, unusual thought content. Similarly, six out of the ten MDARS' s symptoms/signs showed significant $(P<0.05)$ mean rank differences between individual depressive symptoms/signs and the HIV-I seroprevalence: apparent sadness, reported sadness, reduced sleep, inability to feel and suicidal thoughts. It can be seen in Tables 8 and 9 that HIVI seropositive subjects had worst psychiatric symptoms/ signs than HIV-I seronegative subjects. Comparison was made between HIV/AIDS serostatus and gender in relation to depressive symptoms/signs. Table 10 shows associations between psychiatric symptoms/signs and gender. The Likelihood Ratios showed significant associations were found in reported symptoms of apparent sadness for both male $(\mathrm{P}=0.037)$ and female $(\mathrm{P}=0.002)$. However, male subjects were consistently reported symptoms of reported sadness $(\mathrm{P}<0.0001)$, inner tension $(\mathrm{P}=0.030)$, and inability to feel $(\mathrm{P}=0.013)$. Interestingly enough, female subjects reported symptoms of suicidal thoughts $(\mathrm{P}=0.017)$ more than male counter parts $(\mathrm{P}=0.843)$.

\section{DISCUSSION}

In spite of the major efforts by the government and NGOs to prevent the spread of HIV disease, changing human behaviour is a very complex issue worldwide. It is therefore of no surprise that the HIV/ AIDS prevalence rate was very high in this less affuent community. It is even much higher than that found in rural areas in Zimbabwe $(1,2)$. Well, the fact that the community is less affluent in itself is self-explanatory. Again, this high prevalence might be attributed partially to a suggestion (41), that the most important determinant of the spread of HIV is the proportion of men engaging in sexual relations with people other than their spouses, including contact with sex workers and short-term partners. In this study, being female subjects, having single sexual partners and married/cohabited were most likely to be HIV seronegative, this is not in agreement with a study done elsewhere (42). This seems to suggest that marriage could be a protective factor against spread of HIV infection. However, having STDs is significantly associated with HIV infection in a number of reported studies (3), but not in the current study. The prevalence of depression was higher among HIV seropositive subjects $(57.4 \%)$ according to MADRS and as well as $71.3 \%$ of them suffered from psychiatric disorders according to BPRS. These are in agreement with previous studies (8-10). In spite of the fact that many authors used different instruments to investigate psychiatric disorders among HIV/AIDS subjects, still the findings are comparable. On one hand, it is difficult to implicate HIV/AIDS as the only causative factor in the high prevalence of psychiatric disorders in this less affluent community. On the other hand, this high prevalence of psychiatric disorders might be explained by the fact that having HIV/AIDS is scaring, since there is no cure at hand. Other factors though not a focus of the current study, may be playing part such as social isolation, stigma, worrying about the financial support to the family versus treatment of HIV infection can increase vulnerability to psychological stresses. In addition, it is worth noting that in African settings people cannot tolerate the unwarranted restricted sexual activities because of HIV/AIDS this may contribute to more psychological stresses (43). CD4 cells count rank was higher in HIV negative than HIV positive subjects $(\mathrm{P}<0.0001)$. This can be explained by the fact that during the asymptomatic phase of the infection, virus replication and clearance appear to be dynamic processes in which high rates of virus production and infection of $\mathrm{CD}+$ cells are balanced by equally high rates of virus clearance (44). Besides, $24.3 \%$ of our subjects were 
AIDS cases. Although, cognitive impairment was not found among these subjects, as well as reported elsewhere $(16,17)$, this does not mean that cognitive impairments do not occur in HIV/AIDS subjects. The major finding of this study is that there was a moderate prevalence of alcoholic use/misuse among HIV/AIDS subjects $(24.3 \%)$, which is lower than the one found in a similar study among urban cammunity in Harare, $30.5 \%$ (45). This discrepancy can be explained by the fact that this is a less affluent and poor community that might turn to religion rather than alcohol use/misuse for their self-healings. The second explanation is that the sample population is mainly women, and this study proved that a significant number of women $(85.1 \%)$ were HIV seronegative and non-users of alcohol. Besides, most of those who are employed earn wages that are below the poverty datum line (26).

The socioeconomic, political and demographic characteristics of the Epworth community, which unequivocally might have a strong bearing on this moderate relationship between alcohol use misuse and HIV infection. A significant number $(64.3 \%)$ of those who were having HIV/AIDS and had two or more sexual partners used/misused alcohol. This study is in agreement with many studies that found a strong association between alcohol use and unsafe sexual practice among psychoactive substance users $(20,46)$. This can be explained by the fact that common health hazards prevailing in this area were over crowdedness, poor sanitation and this can encourage other unhealthy behaviours (26). In addition, this may lead to frustration, stress and consequently to self -medication with alcohol.

Depressive symptoms/signs such as apparent sadness, reported sadness, reduced sleep and inability to feel are not in agreement with reported symptoms/ signs in a previous study conducted in Zimbabwe (10). However, the two studies agree on reported symptoms/ signs lassitude and suicidal thoughts. It is hard to give a concrete explanation to the high level of psychiatric symptoms/signs in this community. First there is element of poverty, malnutrition and high unemployment rate that may contribute indirectly and enormously to psychological distress. Secondly, persistent traditional means of treating ailment in the face of increasing modern medicine may delay patients benefiting from modern medicine and avert psychiatric symptomatology, as previously reported in Zimbabwe (46). This may lead to the disease becoming chronic and subsequently may lead to an over presentation of clinical symptomatology. The current study concords with the notion (47) that a positive HIV serostatus is associated at most with a modest elevation in suicidal risk. This may be explained by the fact that having HIV/AIDS is scaring, since there is no cure at hand, in addition to social isolation and stigma. It is interesting that a significant number of female subjects reported symptom of suicidal thoughts $(\mathrm{P}=0.017)$ more than male counter parts $(\mathrm{P}=0.843)$. This is expected as males during stress may resort to alcohol use for self-medication as opposed to female subjects.

The clinical implication of the current study is that in any clinical setting HIV infection should be considered as a vital hidden differential diagnosis for organic mental disoders. Prevention and control policies such as early detection and a broad range of biopsychosocial interventions are needed for this community. It is important to note that psychiatric syndromes seen in HIVinfected patients are of multiple and mixed aetiologies e.g., substance-induced and require aggressive screening, diagnosis and treatment. The high prevalence of psychiatric symptoms/signs among this less affluent community indicate a need for rapid interventions. This is because many of these subjects are sexually active, breadwinners of their families and it is crucial to promote safer sexual and health seeking behaviour through public education.

The shortcomings of the current study are many:(a) the findings cannot be generalised to other communities in the country, (b) the using of the two sample populations and two sampling methods for the recruitment, but this has been necessitated by the primary aim of the study, and (c) comparison could have been done between the two sample populations those who claimed to be HIV seropositives and those who did not know about their serostatus) had not a small sample size of HIV seronegative $(n=20)$ among those who claimed to be HIV seropositive. In addition, the small sample size of harmful drinkers, as a result the hazardous and harmful drinkers had to be combined for statistical analysis.

\section{ACKNOWLEDGEMENTS}

We wish to thank MARF-Zimre for funding the study, and many thanks go to those research assistants whose names do not appear in the authorship.

\section{REFERENCES}

1. Niles, C, Nystrom, L., Munjanja, S.P., and Lindmark, G. Symptoms and findings related to HIV in women in rural Gutu District, Zimbabwe. C.A.J.M. 1999; 45:16-20.

2. Tswana, S.A., Nystrom, L., Moyo, S.R., Nzara, M., and Boone, R. The relationship between malaria and HIV. C.A.J.M. 1999; 45:43-45.

3. Colvin, M. and Sharp, B. Sexually transmitted infections and HIV in a rural community in the Lesotho Highlands. Sexual Trans. Infection. 2000; 76:39-42.

4. Wassherheit, J.N. Epidemiological synergy: interrelationship between HIV infection and other sexually transmitted diseases. Sexually Trans. Dis. 1992; 19:61-77.

5. Grosskurth, H., Mosha, F., Todd, J., et al. Impact of improved treatment of sexually transmitted disease on HIV infection in rural Tanzania: randomized controlled trial. Lancet. 1995; 346:530-536.

6. Boisson, E.V. and Rodrigues, L.C. Factors associated with HIV infection are not the same for all women. J. Epidemi. Comm. Health 2002; 56:103-108.

7. Centre For Disease Control and Prevention (CDC). First 
500,000 AIDS cases-United States. Morb. Mortal Wkly Rep. 1995; 44:840-853.

8. Sewell, D.D., Jeste, D.V., Atkinson, J.H., et al. HIV associated psychosis: a study of 20 cases. Amer. J. Psychiatry. 1994; 151:237-242.

9. Ciesla, J.A. and Roberts, J.E. Meta-analysis of the relationship between HIV infection and risk for Depressive Disorders. Amer. J. Psychiatry. 2001; 158:725-730.

10. Sebit, M.B., Chandiwana, S.K., Latif, A.S., et al. Neuropsychiatric aspects of HIV disease progression: Impact or traditional herbs on adult patients in Zimbabwe. Progress in Neuropsychopharmacology and Biology psychiatry. 2002; 26:451-456.

11. Williams, J.B., Rabkin, J.G., Remien, R.H., et al. Multidisciplinary baseline assessment of homosexual men with and without HIV infection II. Standardised assessment of current iifetime psychopathology. Arch. General Psychiatry. 1991; 48:124-130.

12. Robins, L. and Regier, D. Psychiatric disorders in America: the Epidemiological Catchment Area Study. 1991:Pp 53-80. (New York, Free Press).

13. Bartlett, J.G., and Gallant, J.E. Medical Management of HIV infections. Johns Hopkins University Press. Baltimore MD. 2000.

14. Sewell, M.C., Goggin, K.J., Rabkin, J.G., et al. Anxiety Syndromes and symptoms among men with AIDS: a longitudinal controlled study. Psychosomatics. 2000; 41:294 300 .

15. Navia, B.A., Jordan, B.D., and Price, R.W. The AIDS dementia complex. I. Clinical features. Ann Neurol. 1986; 19:517-524.

16. Franzblau, A., Letz, R., Hershman, D., et al. Quantitative neurological and neurobehavioural testing of persons infected with HIV-I. Arch Neurol 1991; 48:263-268.

17. Maj, M., Janssen, R., Starace, F., et al. WHO neuropsychiaric AIDS study. Crosssectional phase 1. Study design and psychiatric findings. Arch. Gen. Psychiatry. 1999; 51:39-49.

18. Centre for Disease Control and Prevention. Continued sexual risk behaviour among $\mathrm{HIV}$-seropositive, drug-using menAtlanta; Washington, DC; and San Juan, Puerto Rico, Morb. Mortal. Wkly Rep. 1996; 45:151-152.

19. Eslebanez, P.E., Russell, N.K., Aguilar, M.D., et al. Women, drugs and HIV/AIDS: results of a multicentre European study. Inter. J. Epidemiology. 2000; 29:734-743.

20. Woods, W.J., Lindan, C.P., Hudes, E.S., et al. HIV infection and risk behaviours in two cross-sectional surveys of heterosexuals in alcoholism treatment. J. Studies on Alcohol. 2000; 61:262-266

21. Lipstiz, J.D., Williams, J.B., Rabkin, J.G., et al. Psychopathology in male and female intravenous drug users with and without HIV infection, Amer. J. Psychiatry, 1994; 151:1662-1668.

22. Batki, S.L., Ferrando, S.J., Manfredi, L.B. et al. Psychiatric disorders, drug use, and medical status in 84 injection drug users with HIV disease, Amer. J. Addiction, 1996; 5:249-258

23. Perry, S., Jacobsberg, L., Card, C.A., et al. Severity of psychiatric symptoms after HIV testing. Amer. J. Psychiatry. 1993; 150:775-779.

24. Pakesch, G., Loimer, N., Grunberger, J., et al. Neuropsychological finding and psychiatric symptoms in HIV-I infected and non-infected drug users, Psychiatry Res., 1992; 41:163-177.

25. Jewett, J.P. and Hecht, F.M. Preventive health cares for adults with HIV infection. J. Vet. Med. Ass. 1993; 268:11441153.

26. The Zimbabwe 1992 census, Central Statistics of Office, Harare, Zimbabwe, 1994.

27. World Health Organization. The Composite International
Diagnostic Interview. (CIDI). 1987, Geneva, Switzerland.

28. American Psychiatric Association. Diagnostic and Statistical Manual of Mental Disorders, Revised Fourth Edition. (DSM-IV). Washington, DC., APA. 1994.

29. Overall, J.E. and Gorham, D.R. The Brief Psychiatric Rating Scale. Psychol. Rep. 1962; 10:799-812

30. Hedlund, J.L. and Vieweg, B.W. The Brief Psychiatric Rating Scale (PBRS): a comprehensive review. J. Oper. Psychiatry. 1980; 11:48-65.

31. Montgomery, Y.S. and Asberg, M. A new depression scale designed to be sensitive to change. Brit. J. Psychiatry. 1979; 134:382-389.

32. Folstein, M.F., Folstein, S., and Mc Hugh, P.R. Mini-MentalState: a practical method for grading the cognitive state of patients for the clinicians. J. Pschiat. Res. 1975; 12:189.

33. Levine, Z.T., Buchanan, R.I., Sekher, L.M., et al. Proposed grading system to predict the extent of resection and outcomes for cranial base meningiomas. Neurosurgery. 1999; 45:221-230.

34. Centre for Disease Control and Prevention (CDC) 1992. 1993 revised classification system for HIV infection and expanded surveillance case definition for AIDS among adolescents and adults. Mrb Mortal Wkly Rep. 1993; 41(RUR-17):1-19.

35. Babor, T.F., De La Fuente, J.R., Saunders, J., and Grant, M. The Alcohol Use Disorders identification test: Guidelines for use in the primary health care.W.H.O Publication No.89.4, Geneva: W.H.O 1989.

36. Saunders, J., Aasland, O., Babor, T.F., et al. Development of the Alcohol Use Disorders Identification Test (AUDIT): W.H.O Collaborative project on early detection of persons with harmful alcohol consumption-II. Addiction, 1993; 88:791-804.

37. Duke, P., Pantelis, C., and Barres, T. South Westminster Schizophrenia's survey: alcohol use and its relationship to symptoms, tardive Dyskinesia and illness onset. Brit. J. Psychiatry. 1994; 184:630-636.

38. World Health Organization. Composite International Diagnostic Interview (CIDI) Training Manual (Draft). Geneva, Switzerland, WHO. 1989.

39. Lisse, I.M., Whittle, H., Aaby, P., et al. Labeling of T cell subsets under field conditions in tropical countries: adaptation of the immuno-alkaline phosphatase staining method for blood smears. J. Immuno Methods. 1990; 129:49-53.

40. WHO Global Programme on AIDS. Laboratory manual for a simple method for characterization of $\mathrm{T}$ cell subsets in peripheral blood: an immune alkaline phosphatase method. WHO: DIA/ MISC/92.2, Rev.1. 1993.

41. Auvert, T.S., Buonamica, G., Lagorde, E., and Williams, B. Sexual behaviour, heterosexual transmission and the spread of HIV in sub-Saharan Africa immulation study. Computers and Biomed. Res. 2000; 33:84-96.

42. Quigley, M.A., Morgan, D., Malamba, S.S. et al. A case-control study of risk factors for incident HIV infection in rural Uganda. J. AIDS. 2000; 23:418-425.

43. Sebit, M.B., Chandiwana, S.K., Acuda, S.W. et al. Quality of life evaluation in patients with HIV-Infection: the impact of traditional medicine in Zimbabwe. Central Afr. J. Med. 2000; 46:208-213.

44. Wei, X., Ghosh, S:K., Taylor, M.E. et al. Viral dynamics in HIV-I infection. Nature. 1995; 373:117-122.

45. Chandiwana, S.K., Sebit, M.B., Acuda, S.W. et al. Alcohol consumption in HIV-I infected persons; a study of immunological markers in Harare, Zimbambwe. Central Afr. J. Med. 1999; 45:303-308.

46. Mutambrirwa, J. Health problems in rural communities in Zimbambwe. Soc Sci. Med. 1989; 29:927-932.

47. O'Dowd, M.A., Biderman, D.J. and Mc Kegney, F.P Incidence of suicidality in Aids and HIV positive patients attending a pyschiatric outpatient programme. Psychosomatics. 1993; 34: 33-40. 